MODELING, IDENTIFICATION AND CONTROL, 1994, VOL. 15, NO. 3, 165-169

doi:10.4173/mic.1994.3.6

\title{
Modeling sustainable development
}

\author{
JACQUES C. J. NIHOUL
}

Keywords: Sustainable development, nested models.

Some of the difficulties of extending economic and ecosystem models to allow for long term forecasting applications to the problem of sustainable development are discussed.

It is argued that the new generation of models required will be nested models with fine time-space grid sub-models of non-global smaller scale phenomena imbedded in global scale coarser grid models. These models will have to be constructed by adding the economic dimension to the already interdisciplinary ecosystem models and will require the introduction of an economic numeraire which is invariant and compatible with common units of the other disciplines.

\section{Introduction}

The concept of sustainable development has received increasing attention in the recent years stimulated by national and international concerns over the interrelationship between biological populations (including man), national resources, environment and economic development.

Sustainable development may be described as 'a pattern of social and structural transformations which optimises the economic and other social benefits available in the present without jeopardising the likely potential for similar benefits in the future' (Gilbert and Braat 1991).

The word sustainable implies a long-term perspective comprising an intergenerational give and take transaction on the use of natural resources for economic development. This requires that a methodology exist to assess present and prospective effects of economic policies on resources and environment as well as the consequences for economy and development of observed and forecast modifications of the resource/environment potential.

There is no doubt that the appropriate methodology is mathematical modeling but the question arises whether existing economic and ecosystem models are truly appropriate for the type of long-term forecasting that sustainable development studies require and how they can be combined to provide the interdisciplinary probe that is needed.

Some aspects of the answer are discussed in the following.

\section{Defaults of economic modeling}

Although we tend instinctively to rely on economics to solve the problem of bringing population and environment issues into development planning, the general feeling is that economic models have not so far been able to meet the challenge.

This failure is commonly attributed to some form of disregard of nature by a science which professes to deal with humans, their activities and their profits.

Received 20 December 1993.

†GHER. University of Liège, Belgium. 
In reality, it is not so much a question of contempt for resource and environment constraints as an incapacity to take into account processes with time-scales extending from years to decades and centuries in a framework conceived to analyse the workings of the market economy. Economic modelers are more or less confronted, with sustainable development, with the same problem as coastal engineers have been when, specializing in tide and storm surge forecasting, they were faced with modeling climate changes.

Moreover, even if there was a willingness to extend conventional economic models to allow for long-term evaluation of economic development programmes, the exercise would inevitably stumble against what is probably the greatest shortcoming of classical economics, viz the use of money as a measure of values.

Despite its obvious utility in day-to-day business transactions, money is nothing but a convention which is permanently manipulated by governments' and banks' monetary strategies.

It is difficult to see how the interdisciplinary models required for the management of sustainable development could rise from the foundations of a science - and it must be the only one of the sort - that uses a numeraire of unknown and changing value in its computation (Gilbert and Braat 1991).

\section{Difficulty of long-term ecosystem modeling}

The present generation of ecosystem models which include a preliminary determination of the basic hydrodynamic and physico-chemical processes (the so-called ecohydrodynamic models in marine science) have reached a stage of interdisciplinary sophistication sufficient to accommodate additional variables describing economic and social development and policies. (Indicative of the efforts in this direction are, for instance, the models of coastal seas, including man as a vulnerable predator and the management of living and non-living resources, developed in the recent years.)

The difficulty with the application of such models to sustainable development is the necessity to carry them over the long-term.

Even if we disregard the problems of available computing power and computing costs (the situation in this respect is continually improving), two main questions remain:

(i) the evolution equations are non-linear and their liability to chaotic solutions sets a limit of predictability to the forecasts;

(ii) independently of the limit of predictability and the vulnerability to slight errors in the initial conditions, in most cases, the mesoscale signal is so large compared to the long-term macroscale one that the latter is not veritably represented (or it is with a $100 \%$ error) in simulation results which mingle the two signals.

It is well established for instance that a single model of (mesoscale) tides and storm surges and (macroscale) residual currents in the North Sea, even if it could be operated for ever, would never provide, in the mean, a satisfactory description of the general circulation which is responsible for the long-term removal of pollutants (Nihoul 1975, 1993).

The answer to the questions above lies in the construction, for long-term predictions, of models describing macroscale quantities defined by averaging the state variables (and their evolution equations) over a time sufficiently large to smooth out the intensive - but naturally versatile and perhaps numerically chaotic - mesoscale components. This procedure is currently applied in marine modeling (Nihoul 1975, 1993). 
There remains however, a problem. As the basic equations are non-linear, the averaging leaves in the mean equations, mean products of deviations around the means, i.e. mesoscale signals. Arguing from the versatility of these signals, we like to see them as a form of mesoscale turbulence and parameterise their effect, extending well documented formulae of turbulence theory. This can be misleading.

Environmental mesoscale processes - and the same is true of economic mesoscale processes - however changing, reversing, and indeed even random they may appear over a large span of time, are always imprinted by some form of organization and coherence which classical parametric formulae for quasi-random sub-grid scale fluctuations cannot take into account. The specificity of the mesoscale signals which remains in the mean products of them, affecting the averaged equations, can significantly modify the long-term solution.

For example, two calculations were made of the general circulation over the North West European Continental Shelf in real atmospheric conditions, for different seasons of the year. In the first one, mean products of mesoscale signals were treated as sub-grid scale fluctuations. In the second case, they were computed explicitly using the results of a preliminary model of tides and storm surges. The first simulation was able to reproduce the broad trends of the general circulation (associated with the inflow and outflow of two branches of the North Atlantic Current) but failed to uncover numerous secondary flows in the form of local gyres, responsible for significant increase, locally, in

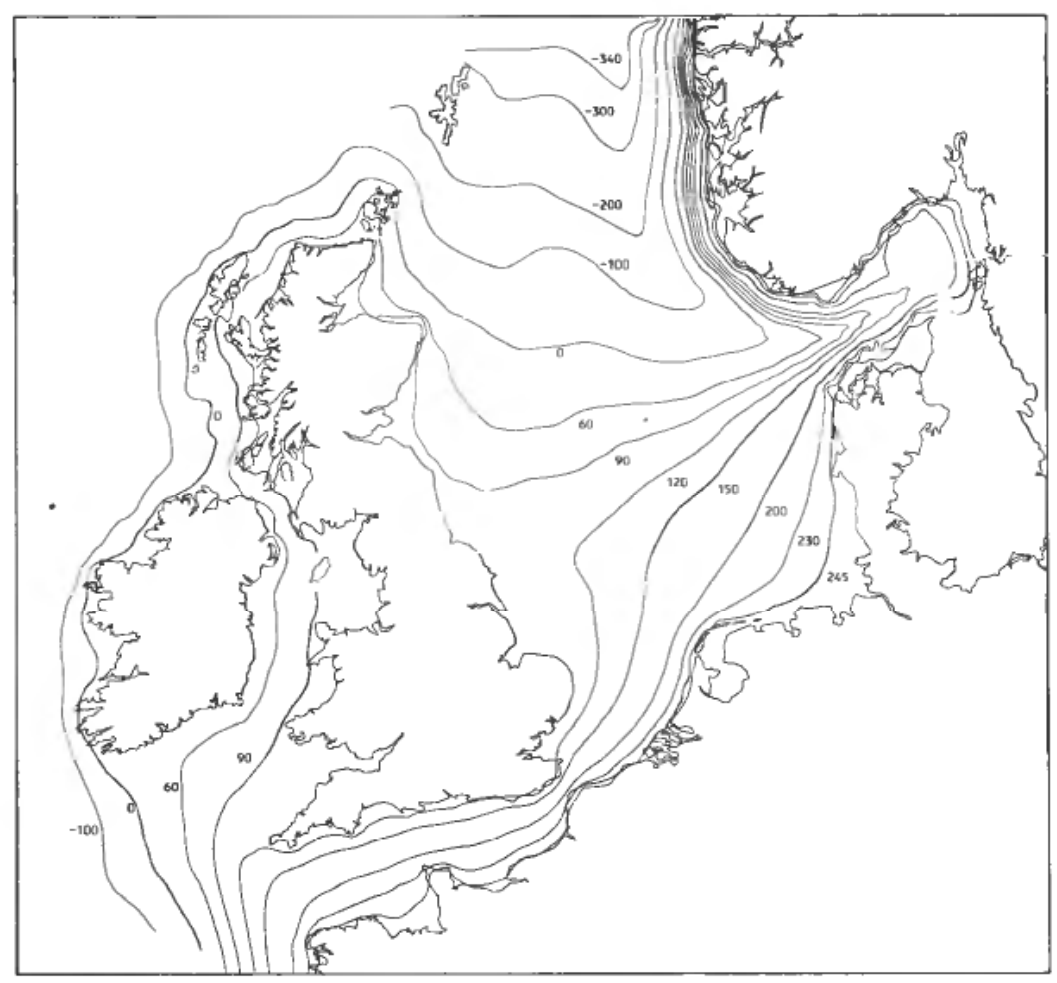

(a) 


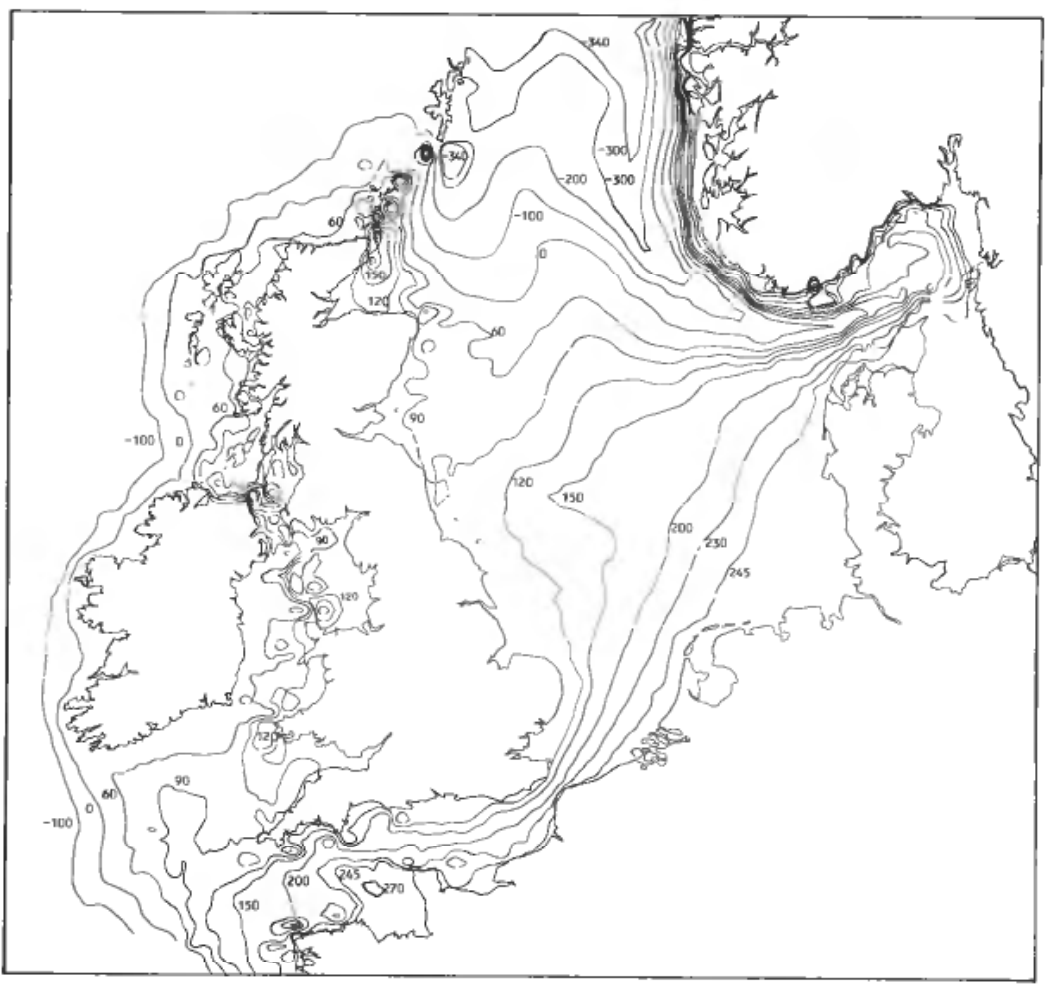

(b)

Figure 1. General circulation over the North-West European Continental Shelf (streamlines of the depth-integrated general circulations graduated in $10^{3} \mathrm{~m}^{3} \mathrm{~s}^{-1}$ ) in a typical summer situation without $(a)$ and with $(b)$ a detailed representation of the mesoscale Reynolds stresses calculated by a preliminary model of mesoscale processes.

residence times and severe modifications of the rate of material transport in the long term (Nihoul 1993, fig. 1).

\section{Nested models}

The example given above is illustrative of the kind of problem long-term forecasting is presented with. Reasonable accurate prediction requires a sufficient understanding of the cumulative effect of medium term, often intense, events from which the environmental, economic and social tissue normally recovers in the long term but which imprint, on global trends, enough of their specificity to produce significant differences.

The same is true, of course, of the role of intense local effects on the global spatial pattern of the system.

One of the conclusions of the workshop held in July 1993 in Villefranche-sur-Mer by the Globec International's Modeling Group, was that the development of ecosystem models, in a perspective of global change and of sustainable development, would require the operation of nested models in space and in time, solving - on finer time and/or space grids - for the details of non-global smaller scale phenomena, in determinant regions of space and/or periods of time, to be able to adequately parameterise the residual effect of their non-linear interactions on the long-term prevision (Globec 1993). 


\section{REFERENCES}

GiLbert, A. J., and BraAt, I. C. (1991). Modelling for population and sustainable development (Routledge Publ., London) $261 \mathrm{pp}$

GloBeC (Global Ocean Ecosystem Dynamics), (1993). Globec International Newsletter, 1, 1-9. NiHoul, J. C. J. (1975). Modelling of marine systems (Elsevier Publ. Co., Amsterdam) 272 pp. NiHOUL, J. C. J. (1993). Application of mathematical modelling to the marine environment. In Environmental Modeling I, edited by P. Zannetti (Computational Mechanics Publ., Southampton) $75-140$. 